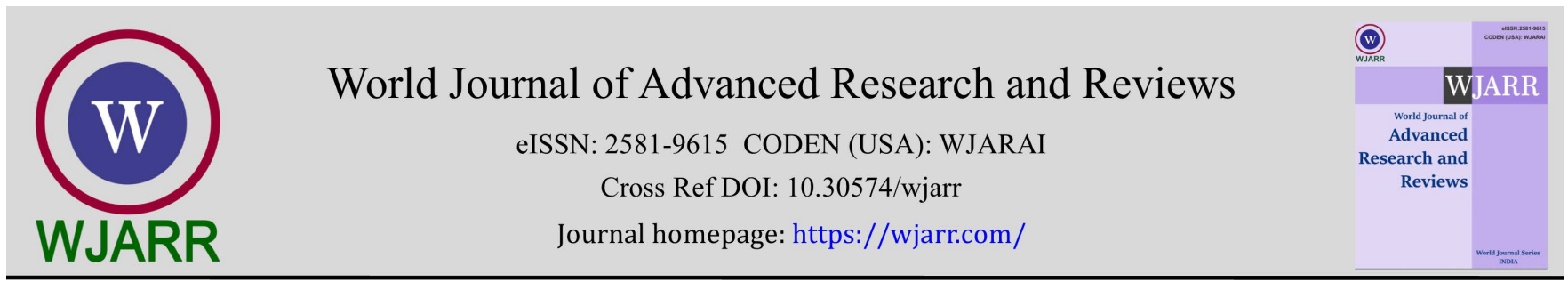

(CASE REPORT)

Check for updates

\title{
Post exenteration surgery rehabilitation with orbital prosthesis - A case report
}

\author{
Ainy Fitri Nuriyanto ${ }^{1, *}$ and Agus Dahlan ${ }^{2}$ and Abil Kurdi ${ }^{2}$ \\ ${ }^{1}$ Resident, Department of Prosthodontics, Universitas Airlangga, Surabaya, Indonesia. \\ 2 Department of Prosthodontics, Universitas Airlangga, Surabaya, Indonesia.
}

World Journal of Advanced Research and Reviews, 2022, 13(01), 072-076

Publication history: Received on 12 November 2021; revised on 28 December 2021; accepted on 30 December 2021

Article DOI: https://doi.org/10.30574/wjarr.2022.13.1.0702

\begin{abstract}
Orbital defects with total loss of eyelids and the eyeball cannot be satisfactorily repaired by reconstructive surgery. Therefore, a prosthetic placement is the treatment of choice to return the individual's confidence by producing an acceptable and lifelike appearance. Male patient, 31 years old, came with history of exenteration surgery four months ago on right eye because of infection few years before. Patient wanted orbital prosthesis to improve esthetic and selfconfidence. He lost his right eye ball and some part of right eyelid. Treatment of choice that would be fabricated was orbital prosthesis with medical adhesive retention support. Orbital prosthesis was one of the alternative that can be chose to reconstruct orbital defect. The success of the prosthesis was determined by the exactness of diagnosis, treatment plan determination, material selection, determination of prosthesis retention, and technique in processing the prosthesis.
\end{abstract}

Keywords: Facial Defect; Orbital Prosthesis; Exenteration; Medicine

\section{Introduction}

Orbital deformities commonly occur from oncological surgery, trauma, or congenital malformation, causing besides loss of vision, other significant restrictions which have a strong impact on the quality of life by negatively affecting aesthetic appearance and restricting social interactions [1]. The loss of an eye through accident, from disease or congenital causes is a major event that impacts on a person's self-image and well-being [2]. As the maxillofacial patient's quality of life is altered and social integration becomes difficult, the patient's expectations to return to "normally" often collapse. The prosthodontist's goal is a successful treatment outcome, but not at the expense of one's emotional and mental wellbeing or that of staff. There must be unconditional commitment to the same treatment goals by both doctor and patient [3].

Prosthetic rehabilitation such as Orbital Prosthetic is an alternative treatment option to surgical reconstruction. These prostheses mimic the patient's missing structures and have acceptable aesthetic outlook. They also permit hygiene maintenance around the defect and observation for tumor recurrence [4].

Although the prostheses do not restore vision, its purpose is to give the appearance of normal facial structure. Unlike other areas of the face that can be adequately repaired by plastic surgery procedures, the orbital region still cannot be recovered by surgical techniques, since only cutaneous covering of extensive cavities can be achieved after tumor resections [1].

These prostheses were attached by means of rubber bands, glasses, or adhesives, but with the advent of osseointegration, there has been a great technical advancement in terms of adaptation, retention, and stability [1].

\footnotetext{
${ }^{*}$ Corresponding author: Agus Dahlan

Department of Prosthodontics, Universitas Airlangga, Surabaya, Indonesia.

Copyright $(2022$ Author(s) retain the copyright of this article. This article is published under the terms of the Creative Commons Attribution Liscense 4.0.
} 
Various types of medical grade adhesive systems are commercially available, such as spray-on adhesives, pastes, double-sided adhesive tapes, and liquid emulsions to retain the prosthesis [4]. Many studies have demonstrated the benefit of implants for retention of the orbital prostheses in providing increased retention, comfort, safety of use, and handling, compared to adhesive or other retention. Considering aspects of public health, with its limited resources and high patient volumes, it is important to evaluate whether the cost-effectiveness is in favor of the use of implants. Consequently, especially in countries with low budget public health systems, determining the use of a specific technique should take into account the economic aspects relative to the impact on patients' QOL related to this procedure [1].

An ideal maxillofacial prosthesis material should have ideal mechanical and physical properties. Methyl methacrylate, polyurethanes, and silicone have gained the most popularity as prosthetic materials. Methyl methacrylate is readily available, durable, and compatible with the tissues, but it is hard, lacks texture, and difficult to match color. Polyurethanes have a high tear strength, superior esthetics, good ultimate strength, and elongation but they are moisture sensitive during curing, resulting bubbles and loss of mechanical properties. Silicone may not be as durable but it is easy to color and has better cosmetic outlook. The major drawbacks of silicone are their low tear and edge strengths and relatively low elongation [4].

This article describes a simplified method for the fabrication of a silicone orbital prosthetics.

\section{Case Report}

A 31-year-old man was referred to the Undaan Eye's Hospital from Soetomo's Hospital with the chief complaint of a missing right eye. A history of exenteration of orbit, which had been carried out due to infection. The defect site consisted of a deep inferior-medial and superior-medial with an intact orbital floor, there is a whole defect in the mesial side (Figure 1).

An ocular prosthesis is manufactured for both ocular and orbital defects, as the orbital prosthesis has an ocular component integrated into it. Diameter of iris is $11 \mathrm{~mm}$, the colour of schlera is stelon self-curing \#3, and the colour of iris is dark brown. The silicone material for orbital prosthesis is RTV type and after evaluation and inspection of the anophthalmic socket and defect region, a design of final prosthesis was made (Figure 1)

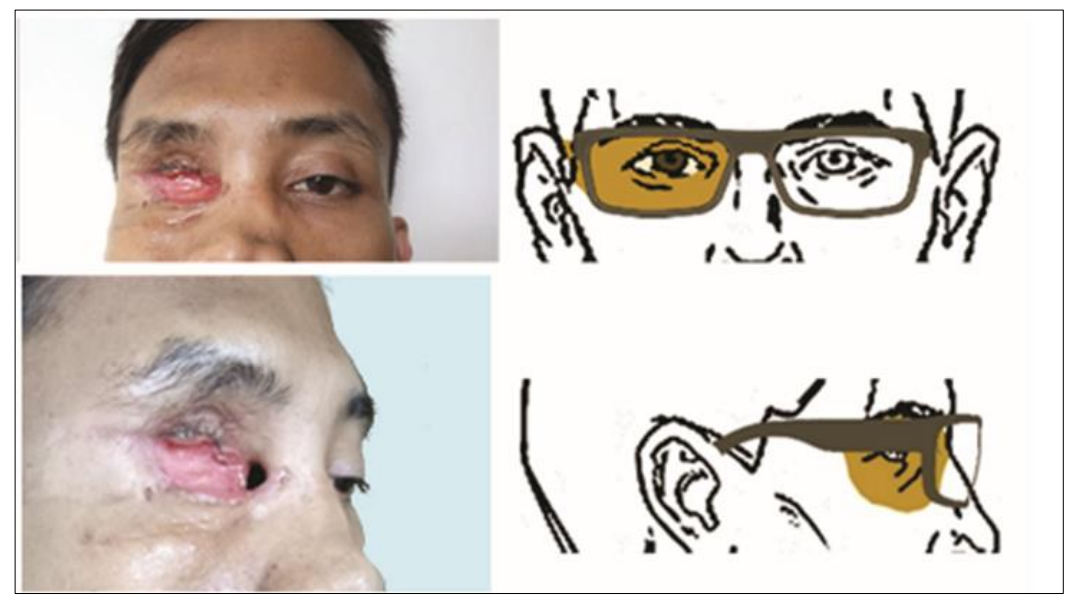

Figure 1 Upper left and lower left: frontal and sagital view of orbital defect from right eye. Right: treatment plan illustration for orbital defect from frontal and sagital view

\subsection{Case Management}

- An informed consent was obtained from patient himself.

- The eyebrow and eyelashes were lightly lubricated using Petroleum Jelly (Nivea, East Africa) so that impression material would not stick on it. Then an impression was made.

- The modelling wax (Cavex, Netherlands) was adapted around the half face to receive the impression material (Figure 2). Direct impression was prepared. Irreversible hydrocolloid (Hygedent, USA) was used, first poured gradually and gently until it covered the entire area, then paper clip were placed over the alginate to hold the dental plaster which acts as a retention for the alginate. The impression material along with dental plaster was 
allowed to set. The set impression was gently taken out in single piece without tearing the impression material (Figure 2).

- $\quad$ The master cast was poured in type II Gypsum (Moldano, USA).

- Next step was making ocular prosthesis first. Size and orientation of the ocular part was determined by taking various measurements on patents contralateral side. Measurements from nasal bridge to the inner canthus, inner canthus to center of iris and center of iris to outer canthus of the contralateral eye were made while patient was looking forward. The measurements were recorded and transferred on to the master cast and

- $\quad$ 'modelling wax was made over the cast and tried in patients face.
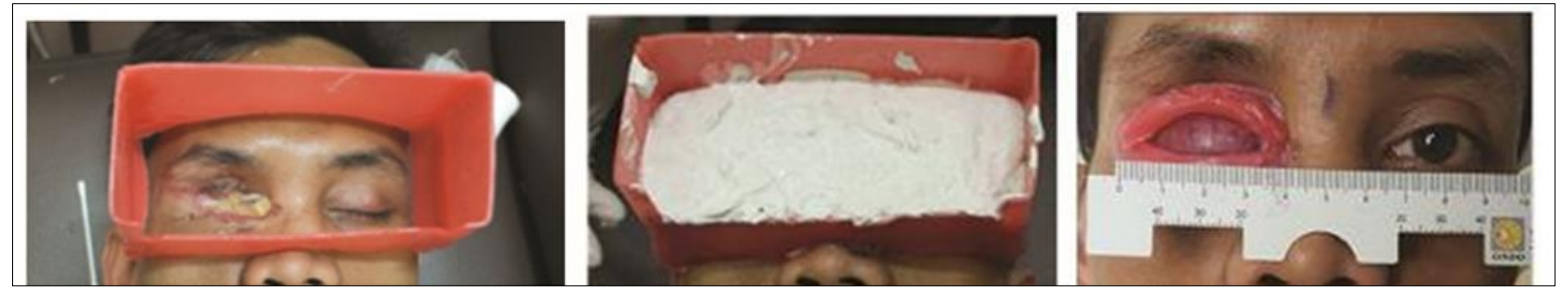

Figure 2 Left to right: Modelling wax was used as a box to make impression. Try-in of modelling wax of sclera shape and iris position

- Sclera pattern was also fabricated with the help of modelling wax. Sclera was tried in patient. And then, processing acrylic (Meliodent Multicryl, Heraeus-kulzer GmbH, Wehrheim, Germany) for occuler prosthesis was done. After that, Iris painting was done to match contralateral eye, blood vessels were placed on the sclera using red embroidery floss, a thin layer of processed clear acrylic resin (Orthocryl, Dentaurum, Germany) was applied over the surface of the painted sclera. Then the ocular prosthesis was trimmed and polished (Figure 3).

- After completing ocular part, the orbital part of prosthesis was fabricated on modeling wax around the defect. The wax prosthesis along with custom made ocular prosthesis was tried on (Figure 3). Patient.eyelids and remaining portion of the prosthesis was carved and tried for final position and fit into defect and to confirmed profile looks (Figure 3).

- $\quad$ The wax pattern was flasked (figure 3) and after boil-out, the temporary base was removed and trimmed 1 mm short of prosthesis border. Mixing room temperature vulcanizing (RTV) (A-2186) silicone with intrinsic coloration was made, the colour must be match with patient's skin. Painting the silicone involves both artistic skills and the science of colour (figure 3)

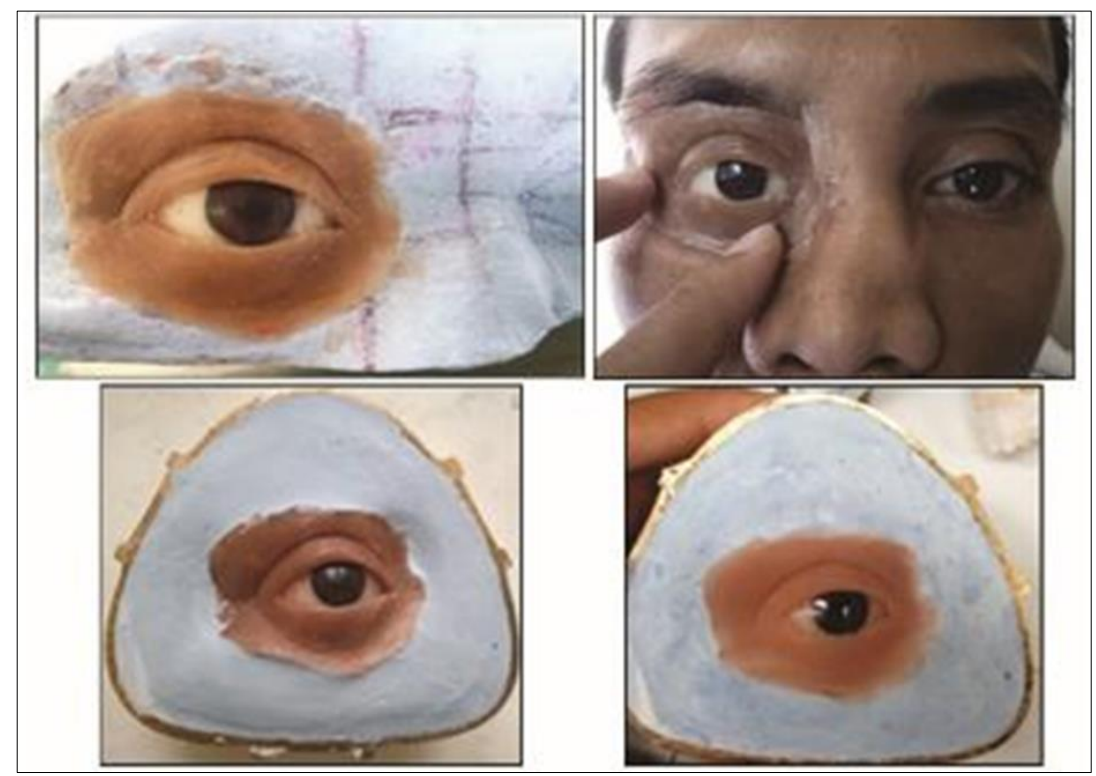

Figure 3 Upper left and right: Carving on modelling wax and try-in of orbital prosthesis Lower left and right: wax pattern was flasked and RTV silicone result 
- Artificial eyelashes implantation was done with hair punching technique (figure 4). Chairside extrinsic coloring for orbital prosthesis was continued using extrinsic coloring sealant. When the sealant already in the setting state, the prosthesis could be inserted to patient (figure 4).

- This prosthesis was attached using glasses (figure 4).

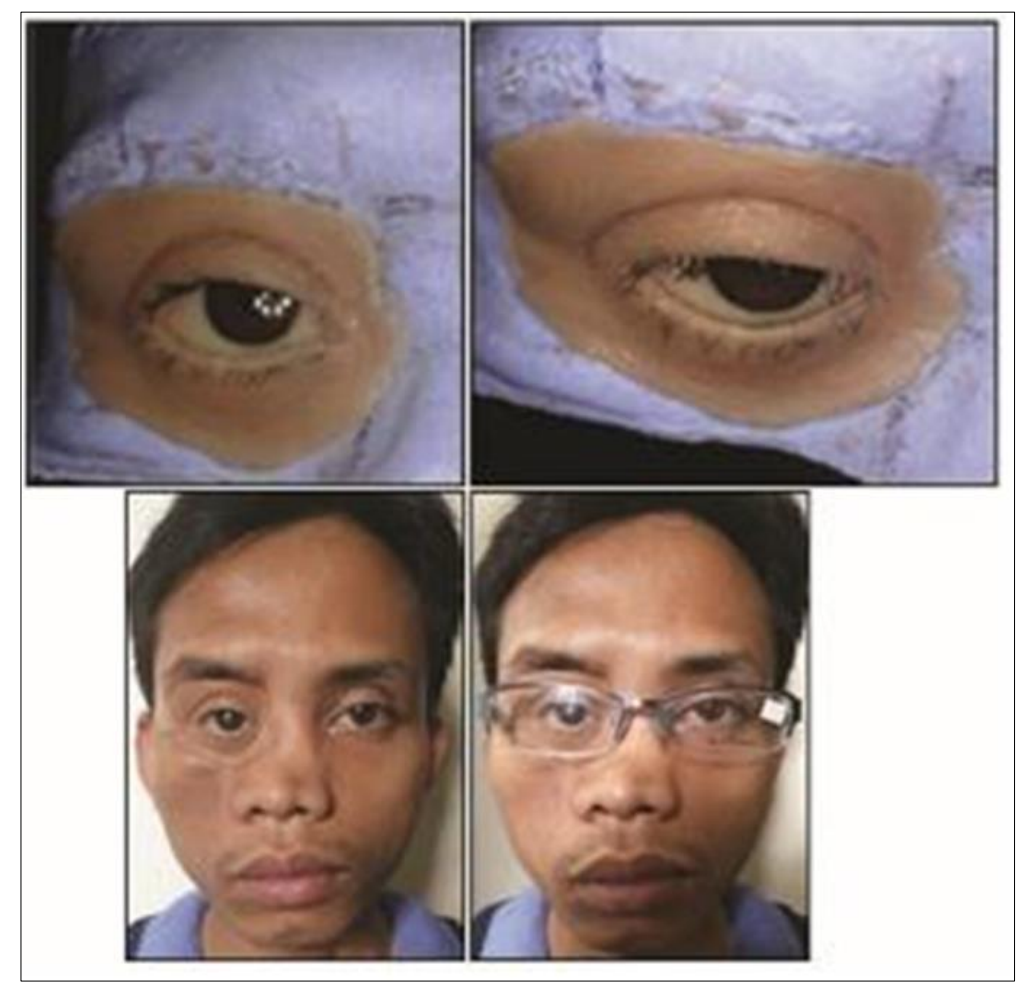

Figure 4 Upper: artificial eyelashes implantation. Lower: prosthesis insertion without glasses, and with glasses for attachment

\section{Discussion}

The prosthesis aims to cover the open cavity after surgery and improve the patient's appearance, psychososial status, and quality of life. Fabrication of a prosthesis for a patients who have lost an eye, tissue around the eye, and nasal is a challenge. After insertion, not just appearance but patient could do various activities like bending, stretching, eating, talking and smiling without the risk of falling or loosening [5].

The availability of anatomic undercut play the major role in the selection of material used for fabrication of the orbital prosthesis. Flexible material will be beneficial for the patients presenting with engageable orbital undercuts. In contrary, in cases with complete loss of orbit and without any possible engageable undercuts, the eyeglass frame retention method is the economical and convenient as eyeglass frames are easier to place in an exact, reproducible prosthesis position [6]. Although, implant-retained ocular prosthesis play an important role in the success of treatment, conventionally retained orbital prostheses are practical, trouble-free, cost-effective and successful [7].

An ideal maxillofacial prosthesis material should have ideal mechanical and physical properties, such as dimensionally stable, high resistance to abrasion, high tear and tensile strength, no water sorption, odorless, it should also be biocompatible with the surrounding tissues without release of any allergenic or toxic by-products after curing [4].

Methyl methacrylate, polyurethanes, and silicone have gained the most popularity as prosthesis materials as they can be intrinsically and extrinsically tinted using oil-based or dry earth pigments to match the patient's skin [4]. Silicone type room temperature vulcanizing (RTV) has better cosmetic outlook because the margins can be made thin and translucent to blend with surrounding skin [4]. 


\section{Conclusion}

Orbital prosthesis was one of the alternative that can be chose to reconstruct orbital defect. The success of the prosthesis was determined by the exactness of diagnosis, treatment plan determination, material selection, determination of prosthesis retention, and technique in processing the prosthesis.

\section{Compliance with ethical standards}

\section{Acknowledgments}

There is no conflict of interest. This study did not receive any financial support and donation.

\section{Disclosure of conflict of interest}

No conflict of interest.

\section{Statement of informed consent}

Informed consent was obtained from all individual participants included in the study.

\section{References}

[1] de Oliveira FM, Salazar-Gamarra R, Öhman D, Nannmark U, Pecorari V, Dib LL. Quality of life assessment of patients utilizing orbital implant-supported prostheses. Clinical Implant Dentistry and Related Research. 2018; 20: 438-43.

[2] Pine KR, Jacobs RJ, Sloan BH. Clinical ocular prosthetics. 2015.

[3] Taylor DT. Clinical Maxillofacial Prosthetics. 1st ed. Illinois: quintessence books. 2000.

[4] Thaworanunta S, Shrestha B. Orbital prosthesis fabrication: current challenges and future aspects. Open Access Surgery. 2016; 21.

[5] Khaghani A, Babaee T, Mohajeri S, Rouhani N. Fabricating A Maxillofacial Prosthesis with A Novel Suspension Technique for A Person with Exenteration: A Case Report. Iranian Red Crescent Medical Journal. 2021; 23.

[6] Singh T, Mathema SRB, Manandhar P. Conventional Approach for Fabrication of Orbital Prosthesis: A Case Report. Birat Journal of Health Sciences. 2018; 3: 495-9.

[7] Hafezeqoran A, Koodaryan R. A technique for fabrication of an orbital prosthesis: a case report. Journal of Dental Research, Dental Clinics, Dental Prospects. 2010; 4: 69-73. 